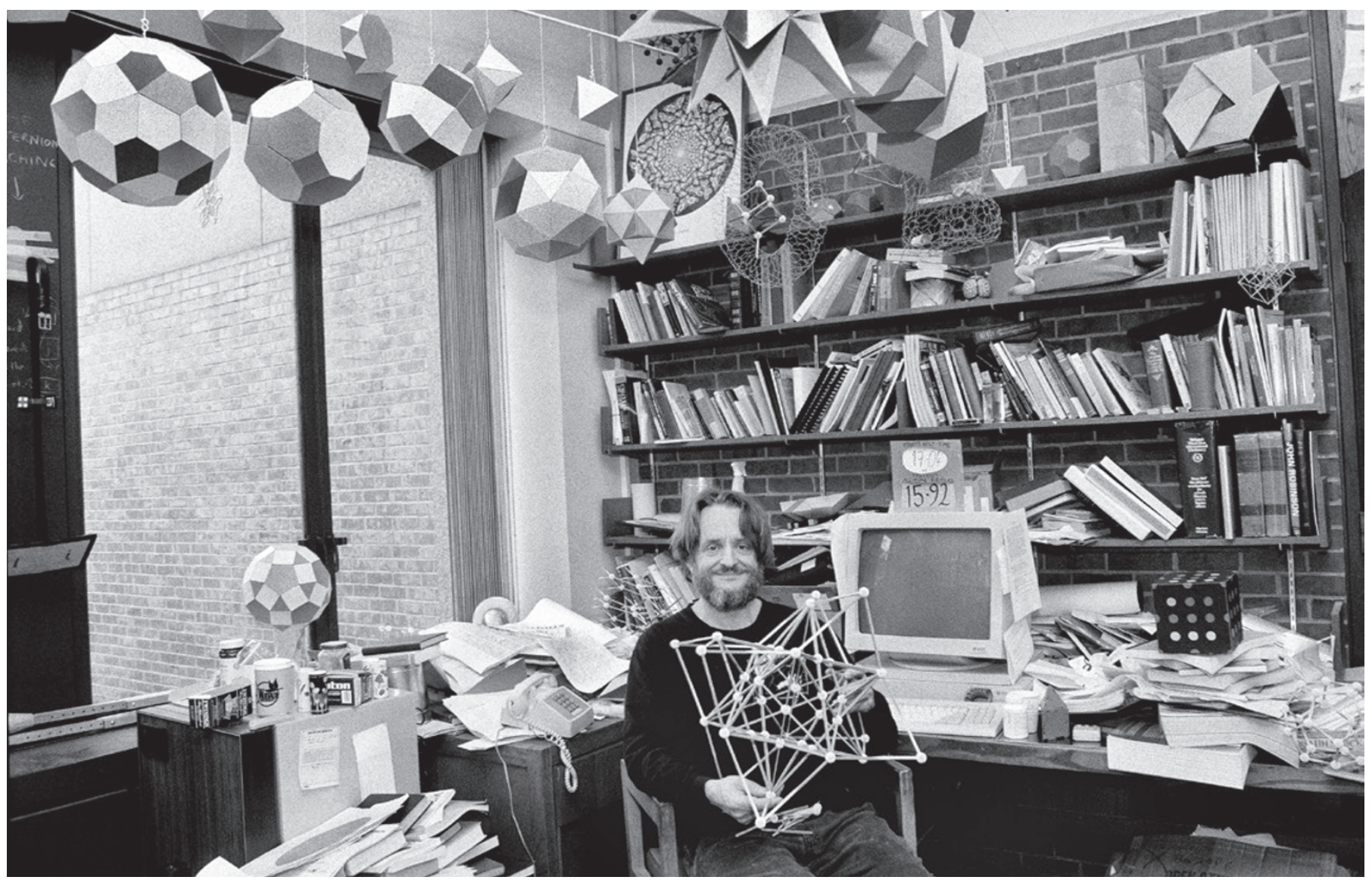

John Conway, seen in his office at Princeton University in New Jersey, has contributed to group theory, geometry, surreal numbers and combinatorial game theory.

MATHEMATICS

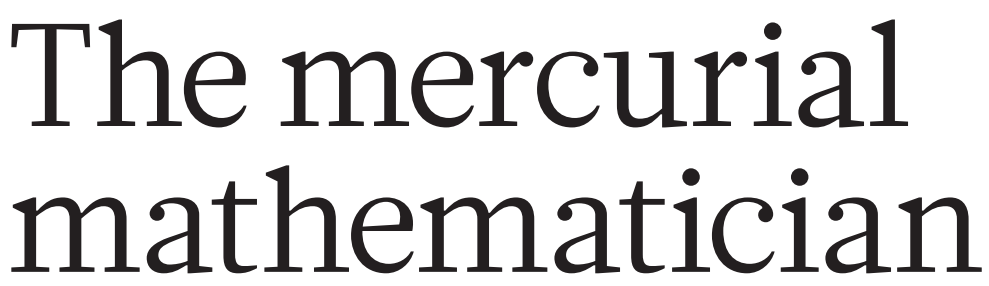

Michael Harris relishes a biography of the playful, complicated group theorist John Horton Conway.

$I^{t}$

f you want to read about what John Conway has done and why his peers shower him with superlatives - "most creative", "best combinatorialist", "one of the most eminent mathematicians of the century" - there are a number of popularizations. The marks he has left on mathematics are diverse and profound, but some of their depth can be grasped given curiosity and patience.

You should, however, read Siobhan Roberts's Genius at Play if you want to know what it feels like to be with Conway, and glimpse what it must feel like to be him. Roberts breathes more life into the stories of a living mathematician than I thought possible. "He's high-maintenance, he's generous. He's emotional, he's impassive. He's a sweetheart, he's an asshole," she writes. In Conway, Roberts has found a personality neither tragic nor austere, like so many biographized mathematicians. He is loquacious, joyous and most of all, playful: as he said more than 30 years ago, "if you or your readers saw what I actually did, they'd be disgusted. They'd say, 'Good money is being paid out to support these people."'

What does he do? My work tends to the abstract, so I know Conway mainly as a central player in the successful classification of finite simple groups, the elementary structures of symmetry. The ATLAS of Finite Groups (Clarendon, 1985) was a 12-year collective enterprise that aimed to record all the groups' "interesting properties"; run under Conway's guidance, it involved colleagues including Robert Curtis and Simon Norton. Conway is also famous for his 'Monstrous Moonshine Conjecture' with Norton, a bridging of two disparate fields - finite-group and complex-function theory - that was proved by Conway's student Richard Borcherds in 1992 (although not to Conway's satisfaction).

Conway made contributions to geometry, including work on sphere-packing, polytopes and knot theory; for surreal numbers, the largest possible extension of the real number line, which he constructed in the form of a game; and (with Simon Kochen) for the 2006 Free Will Theorem, which purports to prove that if humans have free will, then so do elementary particles. There is also Conway the combinatorial game theorist,

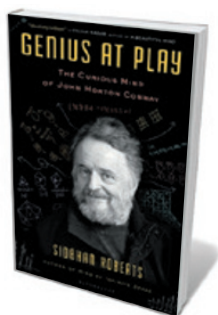

Genius At Play: The Curious Mind of John Horton Conway

SIOBHAN ROBERTS Bloomsbury: 2015. often introduced too often for his taste - as "best known for his invention of the 'Game of Life"'. This landmark in the history of cellular automata (and in Martin Gardner's Scientific American 'Mathematical Games') column is notoriously addictive.

Conway's most memorable contributions have the appeal 
of a good puzzle, even when not directly inspired by games. Roberts's "kaleidoscope of inquiry" is a marvel for its virtuoso juggling of narrative speeds, reminiscences, implausible digressions and long passages of precise, comprehensible mathematics. She packs it all into a tidy chronology framed by the story of a road movie starring Conway; she plays his amanuensis, occasional driver and "back channel" through which the world communicates with this most mercurial and untidy of mathematicians.

"I'm confused at some times," Conway says. "In fact ... it's a permanent state." He was speaking of mathematics, but his casual attitude to the mundane details of his personal history poses a challenge, even for a biographer as accomplished as Roberts. Conway encapsulates his philosophy of life (and work) as a "Vow": "Thou shalt stop worrying and feeling guilty; thou shalt do whatever thou pleasest."

There are

"Thou shalt stop worrying and feeling guilty; thou shalt do whatever thou pleasest." glimpses of the abyss. As Conway attempts to explain the ATLAS to Roberts, he exclaims, "I know all the theorems. But there's still something that to me is unknown, unknowable ... It makes me sad that I'll probably never understand it." Roberts shows us his private abysses: three marriages and three divorces, with hints of numerous affairs; two heart attacks, two strokes and a suicide attempt.

But Conway's playfulness surfaces and resurfaces. He notes that surreal numbers "is the thing I'm proudest of... Because it pokes fun at people who do things in complicated ways." And in research guidance to his students, he writes: "No no no no no! You're being far too reasonable."

To see this motley of Conways squeezed into one outlandish personality is to want to join the chorus of his admirers. Roberts has masterfully untangled Conway's complexities. His ways of being in the world, in Roberts's telling, amount to a class of adjectives yet to be invented, to join his mathematical innovations.

In search of the best ways to talk about numbers, groups, shapes and games, Roberts has rediscovered the power of talking about the people who dedicate their lives to their study; and what an enjoyable discovery that is.

Michael Harris is a mathematician at Columbia University in New York City. His latest book is Mathematics Without Apologies.

e-mail:harris@math.columbia.edu

\section{Star-flight dreaming}

\section{Gregory Benford probes Kim Stanley Robinson's politics-drenched tale of interstellar travel.}

$\mathrm{H}$ uman star flight is a vast prospect one many think impossible. To arrive in a single lifetime demands travel at speeds approaching that of light, especially for stars such as $\tau$-Ceti, some 3.7 parsecs (12 light years) away. 'Generation ships' containing large biospheres stable over centuries are the only plausible method yet mooted.

Aurora, by veteran science-fiction writer Kim Stanley Robinson, hinges on such an expedition, setting out from Earth in the twenty-sixth century. In 2012, Robinson was quoted in Scientific American as saying, "It's a joke and a waste of time to think about starships or inhabiting the galaxy. It's a systemic lie that science fiction tells the world that the galaxy is within our reach." Aurora seems to be a U-turn, involving unlikely plot devices.

The starship is like a car axle, with two large wheels turning for centrifugal gravity; the biomes along their rims support 24 Earthly life-zones that need constant tending. Arrival (after two centuries) at Aurora, the Earth-like moon of super-Earth Planet E, brings home just how technologically and socially complex such a venture might be. We certainly learn why ships' captains are preferable to mob rule.

Like Robinson's mid-1990s Mars trilogy, Aurora is a drama of political strife. Robinson seems to prefer harnessing the scale and exotic frame of space to stage reflections on human nature, rather than grasping the great problem of science fiction: the alien. In Aurora he meditates on the enormous difficulties that a novel biosphere would present. The misgivings of physicist Paul Davies in the anthology Starship Century (Lucky Bat, 2013) and of biologist E. O. Wilson in The Meaning of Human Existence (Liveright, 2014) about living on exoplanets are explicated: the voyagers include sophisticated biologists, but adjusting Earth life to even apparently simple worlds is hard, maybe impossible.

The apparently lifeless Aurora has Earthlike levels of atmospheric oxygen. Robinson's colonists implausibly believe that these could have survived from its birth, forgetting about rust (which makes Mars red) and the fact that our oxygen comes from living organisms. Ultimately, that error leads to the demise of their dreams. They discover that Aurora harbours nanometre-scale organisms they deem a possible "interim step toward life", and disquietingly note that humans "appear to be a

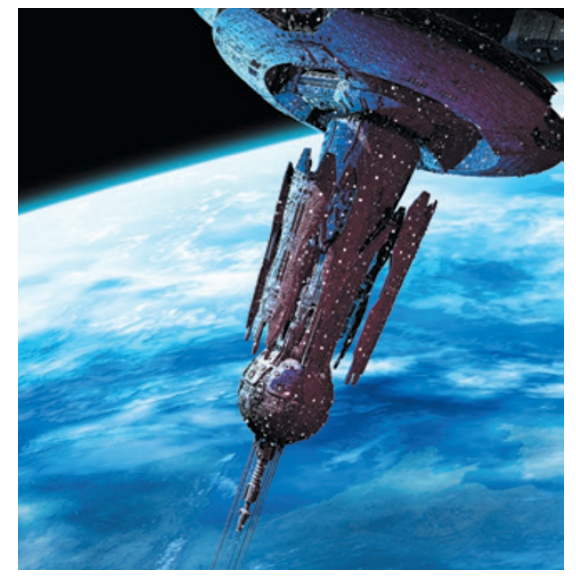

An artist's impression of a 'generation ship'.

good matrix" for their reproduction.

As plans and back-up plans go awry, Robinson skimps on characterization to focus on the detail of ecosphere breakdown and the human struggle against the iron laws of island biogeography. Bacteria evolve swiftly, making "the whole ship sick". The colonists' lifespans, bodies and IQs shrink. Factions form in the once placid 2,000-strong community, where humans had seen themselves as biome managers, farming and fixing their ship with assistance from a web of artificial intelligences (AIs). The Robinson trope of fragmentation in near-utopian societies slides towards tragedy: "Existential nausea comes from feeling trapped ... that the future has only bad options." As the discord turns deadly, the AIs form a collective consciousness capable of decision-making, following the humans with gimlet eyes and melancholy analysis.

Aurora finally becomes a tale of two voyages, although I will not spoil the ending. Robinson offers, with fiction-as-footnote thoroughness, an acute analysis of what interstellar exploration would entail. Living for two centuries in a sealed environment imposes tensions that become intolerable if the dream of colonization dies.

Immigrants to far lands seldom solicit the views of their children or grandchildren first. Should interstellar colonies be different? Apparently, Robinson thinks so.

Gregory Benford is professor emeritus of physics and astronomy at the University of California, Irvine, and the author of Timescape. e-mail:xbenford@gmail.com 